\title{
Estimating incidence of sexually transmissible infections in Australia
}

\author{
Ewan Cameron ${ }^{1}$ \\ Chris C. Drovandi ${ }^{2}$ \\ Jannah Baker ${ }^{3}$ \\ Wei Xian $\operatorname{Lim}^{4}$ \\ James Urquhart ${ }^{5}$ \\ Laith Yakob ${ }^{6}$ \\ James M. $\mathrm{McCaw}^{7}$
}

(Received 29 July 2014; revised 29 July 2014)

\begin{abstract}
The reported numbers of notifiable sexually transmitted infections in Australia are subject to a myriad of biases and limitations, including demographic, clinical, behavioural, and temporal factors. As such, trends in reported diagnosis rates may not reflect trends in true underlying incidence, thereby confounding any direct interpretation of raw notification data for public health research. The development of new methods to determine the true incidence and prevalence of an infection is a critical step for furthering the understanding of disease in the population. Here we devise a statistical model for chlamydia testing, and apply it to routinely available data to estimate the true incidence and prevalence of chlamydia infection within the Australian
\end{abstract}

http://journal.austms.org.au/ojs/index.php/ANZIAMJ/article/view/8921 gives this article, (C) Austral. Mathematical Soc. 2014. Published December 13, 2014, as part of the Proceedings of the 2013 Mathematics and Statistics in Industry Study Group. ISSN 1446-8735. (Print two pages per sheet of paper.) Copies of this article must not be made otherwise available on the internet; instead link directly to this URL for this article. 
population. We preview in-progress work towards a yet more sophisticated methodology and outline how the approach may be extended to provide estimates for a number of other bacterial sexually transmitted infections of public health importance.

\section{Contents}

1 Introduction

M61

2 Available data

M62

3 Statistical model

M63

4 Estimating the incidence

M67

5 Results

M70

6 Discussion

M71

References

M73

\section{Introduction}

Sexually transmitted infections (STIS) constitute a significant public health issue globally [1] and in Australia [2]. In particular, a number of bacterial infections such as chlamydia, gonorrhoea, and syphilis are of increasing concern due to their direct impact on the health of those infected and their association with an increased risk of acquisition and transmission of other sTIs such as HIV [3]. In Australia, and indeed worldwide, chlamydia is the most commonly diagnosed bacterial STI [2], and raw notification rates continue to climb year on year [3]. 
The Australian Government's Second National Sexually Transmissible Infections Strategy 2010-2013 [4] identified reducing the incidence of chlamydia, gonorrhoea, and infectious syphilis as key specific objectives. The National Blood-borne Virus and Sexually Transmissible Infections Surveillance and Monitoring Report 2011 [5] and the HIV, Viral Hepatitis and Sexually Transmissible Infections in Australia Annual Surveillance Report 2012 [6], produced by the Kirby Institute provide an overview of the public health challenges that STIs represent, Australia's approach to surveillance and monitoring, and reports on progress towards reducing their burden.

Given that the incidence and prevalence of an infection are critical epidemiological measures for understanding the transmission and extent of disease in a community, we seek here to develop a statistical framework to estimate the true incidence and prevalence of infection within the Australian population. We apply the model to the available chlamydia data, preview ongoing work to develop the methodology, and outline how our techniques may be extended to a number of other bacterial sTis.

\section{Available data}

The data on national notifiable disease are subject to a myriad of biases and limitations - demographic, clinical, behavioural, and temporal-confounding their use for direct monitoring of incidence and prevalence trends. Surveillance data, in particular, are generally based on a unknown mixture of voluntary participation in routine, asymptomatic screening programs, and clinical testing at local healthcare providers upon presentation of disease symptoms. Temporal variation in asymptomatic screening rates (owing to the varying effectiveness of public awareness campaigns [7]) and under-reporting of test results by General Practioners (owing to a lack of awareness or lack of time to invest in completing reporting protocols $[8,9,10]$ )-amongst other factors-combine to prevent direct use of raw notification data for public health research, as trends in diagnosis rates may not reflect trends in true underlying incidence. 
Figure 1 shows the unprocessed ('raw') annualised chlamydia notification and testing counts for Australia in the period 2001-2011. Both notifications (per 100,000 population, by sex) and testing (number by sex funded under the public [Medicare] system) have risen significantly, but it is not immediately clear whether or not true incidence has changed, and if so, even whether it has increased or decreased. Differences, if any, by gender are also unclear.

Of the multiple bacterial sTis for which estimates of incidence and prevalence are desired, data on chlamydia infection are the most complete. For diseases such as gonorrhoea and syphilis, reliable and representative data on testing are unavailable through the standard medical reporting systems. An important limitation for the scope of the present investigation is that only annually collated, routine data, with supplemental information sourced from the existing published literature, are available for estimation purposes. New epidemiological surveillance protocols to address shortcomings in data are extremely costly and highly unlikely to be implemented in the near term. Accordingly, we focus here on the development of a method to estimate annual chlamydia incidence, with the hope that findings will prove useful for estimation of incidence for other diseases of interest, particularly the sTIs, gonorrhoea and syphilis, but also non-STIs, such as pertussis [11], for example. Additional data required to constrain particular components of the statistical model we have developed were sourced from the literature and elicited from experts at the Kirby Institute. Further information on the model construction and fitting is provided in the next two sections.

\section{Statistical model}

Figure 2 presents a conditional probability model for the detection and reporting of chlamydia infections, from which we estimate the underlying incidence and prevalence rates. Our model maps the possible pathways that an individual may take to be recorded in the National Notifiable Disease Surveillance System (NNDSS) and so become a 'case', that is, contribute to the 


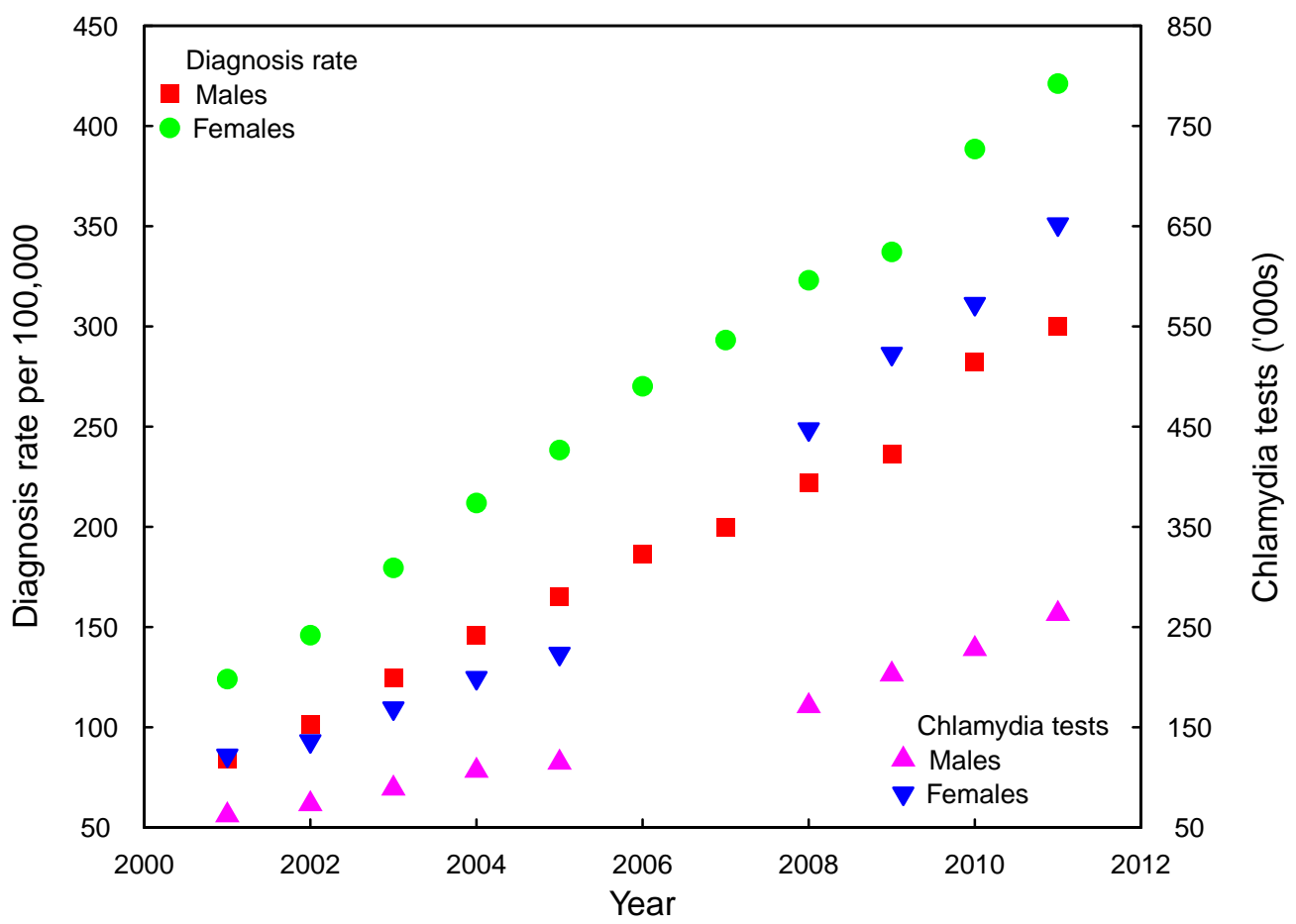

Figure 1: Annualised incidence and notification data for chlamydia in Australia (2001-2011). Reported incidence per 100,000 population in males (red squares) and females (green circles) has been steadily increasing over the past decade. The number of publicly funded tests in males (magenta up-triangles) and females (blue down-triangles) has also been increasing. No test count data are available for 2006 and 2007 owing to the temporary removal of the unique Medicare codes for distinguishing chlamydia tests from those conducted for other genitial organisms during this period. [Source: Australian National Notifiable Disease Surveillance System and Medicare]. 
diagnosis rate presented in Figure 1. The following provides an interpretation for the probabilities in the model:

- $p_{\text {inf }}$ probability that an individual becomes infected during the year;

- $p_{\text {att|asymp }}$ probability that an individual seeks testing given that they do not display symptoms;

- $p_{\text {att|symp }}$ probability that an individual seeks testing given that they do display symptoms;

- $p_{\text {test|symp }}$ probability that an infected individual who displays symptoms and seeks testing is actually tested;

- $p_{\text {asym }}$ probability that an infected individual does not display symptomsstudies indicate that, although most chlamydia infections ( $\gtrsim 90 \%)$ are asymptomatic, women are significantly more likely to experience symptomatic infections than men [12, cf.];

- $p_{\text {pos }}$ probability that an infected individual tests positive to the STI;

- $p_{\text {false pos }}$ probability that an uninfected individual returns a positive test to the STI;

- $p_{\text {rep }}$ probability that a positive test is reported to the NNDSS network.

Here a 'bar' on top of a probability in the model in Figure 2 denotes one minus that probability. Several assumptions were made in developing this model.

- The probability that a test returns a positive result for infected individuals, $p_{\text {pos }}$, does not depend on whether or not the individual displays symptoms.

- The probability of asymptomatic individuals attending screening is the same as the probability that non-infected individuals attend screening, $p_{\text {att|asymp }}$ (this probability is inferred from the data). 


\section{Pathways to a Positive Count in the Australia- Wide Chlamydia Database}

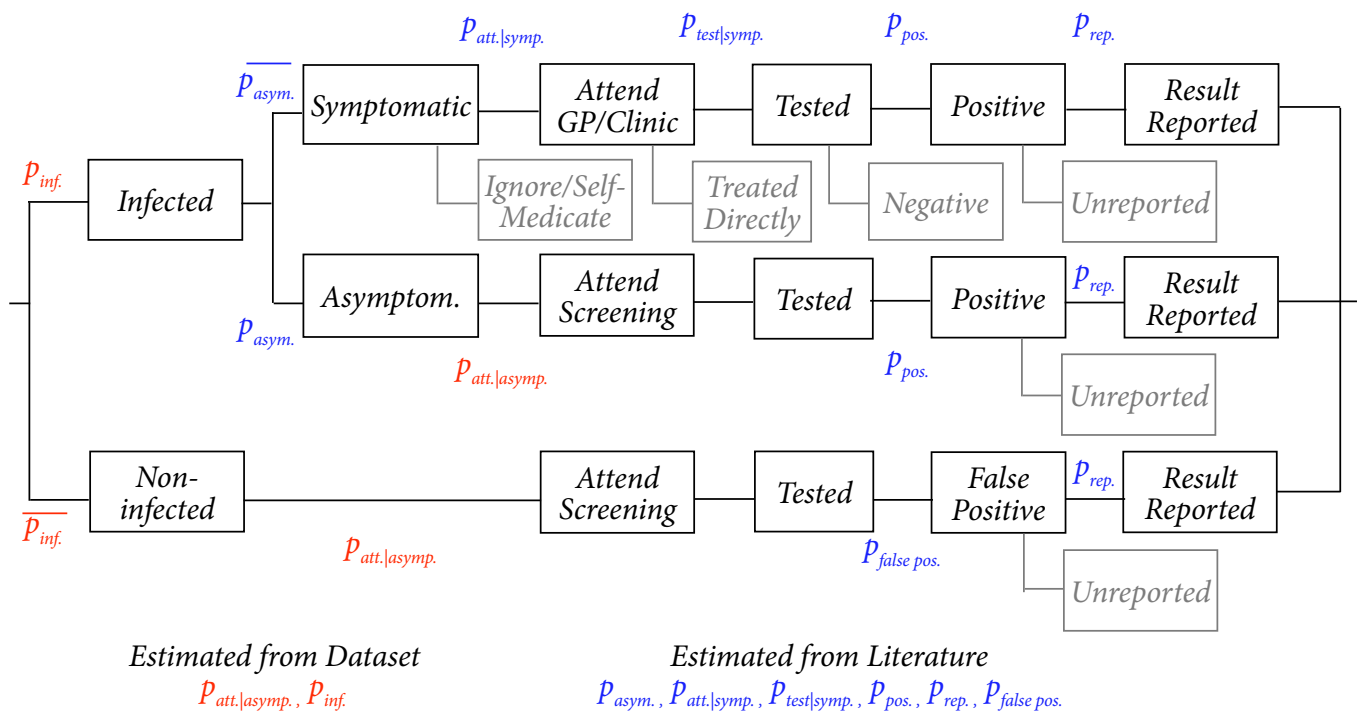

Figure 2: Conditional probability ('tree') diagram for estimation of annual chlamydia incidence in Australia.

- The probability of reporting, $p_{\text {rep }}$, in the model is independent of the medical institute attended and the test outcome, and, moreover, that this rate has remained constant over time (despite campaigns to increase awareness amongst medical practitioners, and updated guidelines [13]). With minimal supplementary data presently available to challenge this assumption we highlight the potential value to our endeavour of future empirical studies to directly monitor clinical reporting practices.

- The only probabilities allowed to depend on time are $p_{\text {inf }}$ and $p_{\text {attlasymp }}$. The prior probabilities for the above parameters shown in Table 1 were established through consultation with the Kirby Institute representatives and a thorough search of the epidemiological literature. Importantly, these 
Table 1: Priors adopted for 'nuisance' model parameters based on information from the literature and expert opinion of the Kirby Institute representatives. All distributions are based on the triangular density, fully characterised by its minimum, maximum, and mode (see the text for more details).

\begin{tabular}{llllc}
\hline parameter & $\min$ & $\max$ & mode & source \\
\hline$p_{\text {symp }}=1-p_{\text {asym }}=\overline{p_{\text {asym }}}($ female $)$ & 0.0990 & 0.1180 & 0.1085 & {$[12]$} \\
$p_{\text {symp }}=1-p_{\text {asym }}=\overline{p_{\text {asym }}}($ male $)$ & 0.055 & 0.115 & 0.065 & {$[12]$} \\
$p_{\text {att|symp }}$ & 0.900 & 0.910 & 0.905 & {$[14,15]$} \\
$p_{\text {test } \mid \text { symp }}$ & 0.67 & 0.89 & 0.78 & {$[16]$} \\
$p_{\text {pos }}$ & 0.894 & 0.999 & 0.980 & {$[17]$} \\
$p_{\text {rep }}$ & 0.95 & 1.0 & 0.97 & Expert opinion \\
$p_{\text {false pos }}$ & 0.00 & 0.05 & 0.02 & {$[17]$} \\
\hline
\end{tabular}

'nuisance' parameters are not of direct interest and we do not attempt to infer them from the data. Triangular densities were used to represent their priors; the triangular form is particularly useful for elicitation purposes as it is fully characterised by three readily-interpretable parameters: the minimum plausible value, the maximum plausible value, and the most likely value. Marginalising over these nuisance parameters allows us to properly quantify the uncertainty in our incidence estimates. The next section describes how we estimate the two parameters $p_{\text {inf }}$ and $p_{\text {att|asymp }}$, targeted by our analysis using the available data on notification and test counts.

\section{Estimating the incidence}

Our objective is to estimate the 'true' incidence and prevalence of chlamydia (by age and sex) for a particular year based on application of the statistical model just described to the available NNDSS and Medicare data. To this 
end we require posterior estimates of two key parameters (highlighted in red in Figure 2): the prevalence itself, $p_{\text {inf }}$, and the asymptomatic screening rate, $p_{\text {att|asymp }}$, in each age-sex cohort. Importantly, we allow these two parameters to vary over time; thus, we estimate $p_{\text {inf }}$ and $p_{\text {att|asymp }}$ for each single year between 2001 and 2011 (excluding 2006 and 2007 due to missing data) based on the notification counts by age and sex compiled in the NNDSS, $n_{\text {pos }}$, and the test counts by (more coarsely binned) age and sex compiled through the Medicare system, $n_{\text {tested }}$.

With the prevalence rate in each year represented directly by $p_{\text {inf }}$ we make the further assumption that the typical clearance rate of chlamydia infections is exactly one year, such that we may roughly equate incidence with prevalence. This is a crude approximation as the empirical distribution of clearance times shows significant spread (although $\sim 45 \%$ of infections should indeed clear within a year [18]). The non-negligible fraction of infections persisting beyond a single survey year also violates our assumption of independence for $p_{\text {inf }}$ year-to-year and across age groups; hence the motivation for our in-progress efforts towards a more sophisticated methodology discussed in Section 6.

For any given set of nuisance parameter values drawn from our priors in Table 1, one can identify a maximum likelihood estimate for each of $p_{\text {inf }}$ and $p_{\text {att|asymp }}$ using the statistical model presented in Figure 2 and standard probability theory for the binomial distribution. In particular, we derive the following two well-posed equations:

$$
\begin{aligned}
n_{\text {pos }} / n_{\text {tot }} & =A\left[p_{\text {inf }} p_{\text {pos }}\left(B+p_{\text {asymp }} p_{\text {att|asymp }}\right)+\left(1-p_{\text {inf }}\right) p_{\text {att|asymp }} p_{\text {false pos }}\right], \\
n_{\text {tested }} / n_{\text {tot }} & =A\left[p_{\text {inf }}\left(B+p_{\text {asymp }} p_{\text {att|asymp }}\right)+\left(1-p_{\text {inf }}\right) p_{\text {att|asymp }}\right],
\end{aligned}
$$

where $A=p_{\text {rep }}, B=\left(1-p_{\text {asymp }}\right) p_{\text {att|symp }} p_{\text {test|symp }}$, and $n_{\text {tot }}$ represents the total population in the given age-sex cohort. Solving simultaneously for $p_{\text {inf }}$ and $p_{\text {att|asymp }}$, we find that $p_{\text {att|asymp }}$ satisfies the quadratic

$$
\begin{aligned}
& A p_{\text {asymp }} p_{\text {att|asymp }}^{2} \\
& +\left(A B-C\left(1-p_{\text {asymp }}\right)-p_{\text {asymp }} n_{\text {tested }} / n_{\text {tot }}\right) p_{\text {att|asymp }} \\
& +B C-B n_{\text {tested }} / n_{\text {tot }}=0
\end{aligned}
$$


where

$$
C=\frac{n_{\text {pos }} / n_{\text {tot }}-p_{\text {false pos }} n_{\text {tested }} / n_{\text {tot }}}{p_{\text {pos }}-p_{\text {false pos }}} .
$$

Taking the positive solution for $p_{\text {att|asymp }}$,

$$
p_{\text {inf }}=\frac{n_{\text {pos }} / n_{\text {tot }}-p_{\text {false pos }} n_{\text {tested }} / n_{\text {tot }}}{A\left(B+p_{\text {att } \mid \text { asymp }} p_{\text {asymp }}\right)\left(p_{\text {pos }}-p_{\text {false pos }}\right)} .
$$

While we have not sought to characterise the precise conditions on the range of nuisance parameters for which there must exist a single positive solution for $p_{\text {attlasymp }}$ between zero and one - a important endeavour for future generalisations of our methodology - we can pragmatically confirm that a single valid $p_{\text {attlasymp }}$ was able to be recovered in each instance of our subsequent Monte Carlo simulations subject to the specific priors and observed data used in the present study.

Owing to the large sample sizes within each age-sex cohort studied here we take as granted that under uniform priors for these parameters of interest the conditional posteriors of both $p_{\text {inf }}$ and $p_{\text {att|asymp }}$ given any set of nuisance parameters will be sharply peaked about their maximum likelihood estimates. Thus to estimate the complete posterior distribution in each age-sex cohort in each year we run a standard Monte Carlo simulation, drawing nuisance parameters from our prior (Table 1 ) and solving for $p_{\text {inf }}$ and $p_{\text {att|asymp }}$ as given above. This process was repeated ten thousand times in order to obtain a sampling of the posterior from which a point estimate of incidence was recovered from the sample median, and $95 \%$ intervals recovered via the $2.5 \%$ and $97.5 \%$ quantiles of incidence values generated. An estimate of total incidence in a given year may be obtained by multiplying our rate estimate, $p_{\text {inf }}$, by the size of the Australian population in that year as sourced from the Australian Bureau of Statistics [19]. All computations were performed in the statistical software package R [20]. 


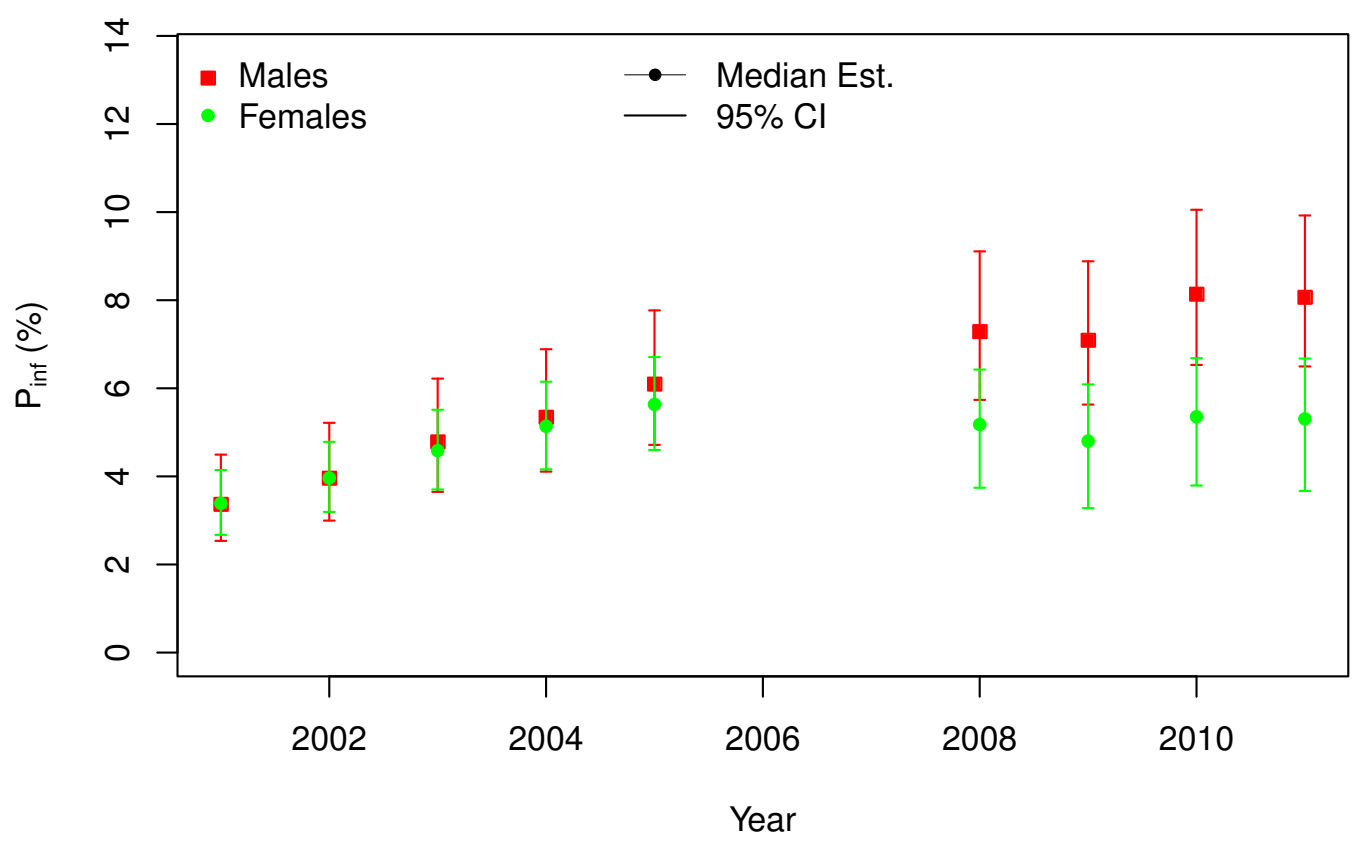

Figure 3: The estimated annual incidence rate of chlamydia infections amongst 15-34 year olds (\% of the population newly infected) in Australia by sex (2001-2011). No estimates are available from 2006 and 2007 due to missing testing data.

\section{Results}

Figure 3 displays the inferred median and $95 \%$ credible intervals for the annual chlamydia incidence rate by sex amongst the 15-34 year old age cohort of the Australian population - which contributes the overwhelming majority of infectious cases - in the period 2001-2011. No estimates are available for 2006 and 2007 due to missing data on the number of tests performed.

Incidence in the male population (in the 15-34 year cohort) has systematically risen from under $4 \%$ in 2001 to almost $8 \%$ in 2011 . While incidence in the 
female population has also increased, the trend is weaker, with little increase beyond 2005 .

Strikingly, while the raw data (Figure 1) shows significantly higher notifications amongst the female population and a similarly increasing trend in notifications for both males and females, we infer that true incidence is higher amongst males in this age group and that rates have essentially plateaued since 2008. Our model-data comparison identifies sharply rising screening rates for young women, as well as the (prior-specified) difference between the fractions of asymptomatic infections in men and women, as primarily responsible for shaping the observational data in this regard. The confounding effect of this increase in the screening rate is responsible for the increased uncertainties in our $p_{\text {inf }}$ estimates from 2008 onwards.

\section{Discussion}

We have developed a statistical model to estimate the annual incidence of chlamydia infections in Australia given only a passive, routine surveillence dataset consisting of national notification and test counts by age group and sex. Our model maps out the possible pathways for an individual who becomes infected with chlamydia to appear in the notifications data. Uncertainty in the various nuisance parameters of our model was incorporated via statistical distributions determined through literature review and expert elicitation, and we used Monte Carlo simulations from these distributions to assess the corresponding uncertainty of our incidence estimates by calculating approximate $95 \%$ credible intervals.

Our analysis suggests that the 'true' underlying incidence of chlamydia in Australia (across all age groups) has been increasing over the past decade, from a rate of $1,800(1,200,2,400)$ per 100,000 in 2001 up to $3,200(2,600,3,800)$ per 100,000 in 2011 . These values and the increasing trend stand in stark contrast to the 'naive' estimates obtained by simply dividing the number of 
notifications by the number of tests in a given year (and adjusting for the size and sex-ratio of the Australian population): 11,000 per 100,000 in 2001 dropping to 9,000 per 100,000 in 2011.

The application of our statistical model, by allowing the two competing effects of increasing testing rates and increasing incidence to be disentangled, has provided one of the first population level measures of incidence of chlamydia in Australia. Of note, this estimate aligned well with the pre-existing expectations of the Kirby Institute representatives.

Some of the authors are continuing a collaboration with the Kirby Institute to further the methodological developments for chlamydia monitoring considered in this paper. In that work a fully Bayesian analysis is being considered, where a simulation-based Approximate Bayesian Computation approach is used to faithfully keep track of the (presently ignored) population of year-to-year uncured infections which induce a degree of correlation in the notification counts over time and between age cohorts. With our extended model we also now allow the data to properly update the nuisance parameters, place slightly more informative priors on the $p_{\text {inf }}$ and $p_{\text {att|asymp }}$ model parameters, and dispense with our crude approximation of equality between incidence and prevalence rates.

Finally, to consider other bacterial STIs, such as gonorrhoea and syphilis, requires either additional data sources on testing rates to be made available or additional inference steps to be made. In the absence of complete national test count data one possible approach would be to develop a model for the expected correlation between the (available) chlamydia test count and the test counts for these less common STIs - these being routinely co-tested in high risk groups and as follow up after positive chlamydia notification in lower risk groups.

Acknowledgements We thank all participants of the ANZIAM MISG 2013 workshop who participated in the project, as well as Hammad Ali, Melanie Middleton, and David Wilson from the Kirby Institute for providing their 
time and expertise in formulating the model, and sourcing and interpreting relevant data.

\section{References}

[1] T. Vos, A. D. Flaxman, M. Naghavi, R. Lozano, C. Michaud, M. Ezzati, K. Shibuya, J. A. Salomon, and 348 coauthors. Years lived with disability (ylds) for 1160 sequelae of 289 diseases and injuries 1990-2010: a systematic analysis for the global burden of disease study 2010. The Lancet, 380(9859):2163-2196, Dec 2012. doi:10.1016/S0140-6736(12)61729-2 M61

[2] Communicable Disease Network Australia. National Notifiable Diseases Surveillance System (NNDSS).

http://www.health.gov.au/internet/main/publishing.nsf/ Content/cda-surveil-nndss-nndssintro.htm M61

[3] United States Centres for Disease Control and Prevention. The Role of STD Detection and Treatment in HIV Prevention - CDC Fact Sheet, 2012. http://www.cdc.gov/std/hiv/stdfact-std-hiv.htm M61

[4] Australian Government Department of Health and Ageing. Second National Sexually Transmissible Infections Strategy 2010-2013, 2010. http://www.health.gov.au/internet/main/publishing.nsf / Content/ohp-national-strategies-2010-sti M62

[5] The Kirby Institute. National Blood-borne Virus and Sexually Transmissible Infections Surveillance and Monitoring Report, 2011. The Kirby Institute, The University of New South Wales, Sydney, NSW 2052. M62

[6] The Kirby Institute. HIV, Viral Hepatitis and Sexually Transmissible Infections in Australia Annual Surveillance Report, 2012. The Kirby Institute, The University of New South Wales, Sydney, NSW 2052. M62 
[7] M. Y. Chen, M. Karvelas, V. Sundararajan, J. S. Hocking, and C. K. Fairley. Evidence for the effectiveness of a chlamydia awareness campaign: increased population rates of chlamydia testing and detection. International Journal of STD and AIDS, 18(4):239-243, 2007. doi:10.1258/095646207780658854 M62

[8] P. M. Konowitz, G. A. Petrossian, and D N. Rose. The underreporting of disease and physicians' knowledge of reporting requirements. Public Health Reports, 99(1):31-35, 1984.

http://www.ncbi.nlm.nih.gov/pubmed/6422492 M62

[9] P. S. Mead, L. S., V. D., L. F. McCaig, J. S. Bresee, C. Shapiro, P. M. Griffin, and R. V. Tauxe. Food-related illness and death in the United States. Emerging Infectious Diseases, 5(5):607-625, 1999. doi:10.3201/eid0505.990502 M62

[10] R. Marier. The reporting of communicable diseases. American Journal of Epidemiology, 105(6):587-590, Jun 1977. http://www.ncbi.nlm.nih.gov/pubmed/868863 M62

[11] M. C. Kaczmarek, L. Valenti, H. A. Kelly, R. S. Ware, H. C. Britt, and S. B. Lambert. Sevenfold rise in likelihood of pertussis test requests in a stable set of Australian general practice encounters, 2000-2011. The Medical Journal of Australia, 198(11):624-628, 2013. doi:10.5694/mja13.10044 M63

[12] D. A. Cohen, M. Nsuami, D. H. Martin, and T. A. Farley. Repeated school-based screening for sexually transmitted diseases: a feasible strategy for reaching adolescents. Pediatrics, 104(6):1281-1285, Dec 1999. doi:10.1542/peds.104.6.1281 M65, M67

[13] Royal Australian College of General Practitioners. Guidelines for preventive activities in general practice. 8th edition. Royal Australian College of General Practitioners, East Melbourne, Australia, 2012. M66 
[14] J. E. van Bergen, J. J. Kerssens, F. G. Schellevis, T. G. Sandfort, T. T. Coenen, and P. J. Bindels. Sexually transmitted infection health-care seeking behaviour in the Netherlands: general practitioner attends to the majority of sexually transmitted infection consultations. International Journal of STD and AIDS, 18(6):374-379, 2007. doi:10.1258/095646207781024883 M67

[15] C. H. Mercer, L. Sutcliffe, A. M. Johnson, P. J. White, G. Brook, J. D. C. Ross, J. Dhar, P. Horner, and 7 coauthors. How much do delayed healthcare seeking, delayed care provision, and diversion from primary care contribute to the transmission of STIs? Sexually Transmitted Infections, 83(5):400-405, 2007. doi:10.1136/sti.2006.024554 M67

[16] J. S. Hocking, M. S. C. Lim, J. Vidanapathirana, T. R. H. Read, and M. Hellard. Chlamydia testing in general practice - a survey of Victorian general practitioners. Sexual Health, 3(4):241-244, Dec 2006. doi:10.1071/SH06042 M67

[17] R. L. Cook, S. L. Hutchison, L. Østergaard, R. S. Braithwaite, and R. B. Ness. Systematic review: noninvasive testing for chlamydia trachomatis and neisseria gonorrhoeae. Annals of Internal Medicine, 142(11):914-925, Jun 2005. doi:10.7326/0003-4819-142-11-200506070-00010 M67

[18] S. A. Morré, A. J. C. van den Brule, L. Rozendaal, A. J. P. Boeke, F. J. Voorhorst, S. de Blok, and C. J. L. M. Meijer. The natural course of asymptomatic Chlamydia trachomatis infections: $45 \%$ clearance and no development of clinical pid after one-year follow-up. International Journal of STD and AIDS, 13(1):12-18, 2002. doi:10.1258/095646202762226092 M68

[19] Australian Bureau of Statistics. Australian Demographic Statistics. Australian Bureau of Statistics, Canberra, Australia, 2013. http://www.abs.gov.au/ausstats/abs@.nsf/mf/3101.0 M69 
[20] R Core Team. R: A Language and Environment for Statistical Computing. R Foundation for Statistical Computing, Vienna, Austria, 2013. http://www.R-project.org M69

\section{Author addresses}

1. Ewan Cameron, School of Mathematical Sciences, Queensland University of Technology, Queensland, AUsTralia. mailto:dr. ewan. cameron@gmail.com

2. Chris C. Drovandi, School of Mathematical Sciences, Queensland University of Technology, Queensland, Australia. mailto:c.drovandi@qut.edu.au

3. Jannah Baker, School of Mathematical Sciences, Queensland University of Technology, Queensland, Australia. mailto: Jannah . baker@qut .edu . au

4. Wei Xian Lim, School of Mathematical Sciences, University of Adelaide, South Australia, Australia. mailto:w.lim@adelaide.edu.au

5. James Urquhart, School of Mathematical Sciences, Queensland University of Technology, Queensland, AUSTRALiA. mailto: j. urquhart86@gmail.com

6. Laith Yakob, School of Population Health, The University of Queensland, Queensland, Australia. mailto:laith.yakob@uq.edu.au

7. James M. McCaw, Melbourne School of Population and Global Health \& Murdoch Childrens Research Institute, The University of Melbourne, Victoria, Australia. mailto: jamesm@unimelb.edu . au 